\title{
Englischer National Health Service vor größter Reform seit 60 Jahren
}

\section{Polarisierte Diskussion über Reform des NHS}

\section{LEONHARD HAJEN}

Prof. Dr. Leonhard Hajen ist Hochschullehrer für Volkswirtschaftslehre an der Universität Hamburg, Fakultät für Wirtschafts- und Sozialwissenschaften, Fachbereich Sozialökonomie

\author{
Im März 2012 ist eine Gesundheitsreform für den \\ englischen National Health Service (NHS) beschlossen \\ worden, die die bisherigen Strukturen radikal \\ verändert. Ziele der konservativ-liberale Regierung \\ sind Bürokratieabbau und Effizienzsteigerung. Ob diese \\ Ziele erreicht werden, ist mehr als zweifelhaft. Vielmehr \\ könnten neue Bürokratien an die Stelle der alten treten. \\ Die neu eingeführte Budgetverantwortung für die \\ Zusammenschlüsse von niedergelassenen Ärzten birgt \\ zudem die Gefahr, dass Risikoselektion betrieben und \\ der Gleichheitsanspruch des NHS aufgegeben wird. \\ Die Reformen sollen über Produktivitätssteigerungen \\ im NHS Einsparungen finanzieren. Dieses Ziel ist \\ ohne Leistungseinschränkungen nicht erreichbar.
}

Großbritannien ist im Vergleich zu anderen europäischen Ländern in der komfortableren Lage, dass es sich in einer Währung verschulden kann, die es selber herausgibt, deshalb steht es nicht im Fokus der Finanzkrise, die einige Länder der Währungsunion an die Grenze der Zahlungsfähigkeit gebracht hat. Darüber wird häufig übersehen, dass sich Großbritannien seit Beginn der Finanzkrise in 2008 gemessen an den negativen Wachstumsraten des Sozialproduktes länger und tiefer in der Krise befindet als zur Zeit der Großen Depression zu Beginn der dreißiger Jahre des letzten Jahrhunderts. ${ }^{1}$ Die Defizite der öffentlichen Haushalte wuchsen und schon die Labour Regierung wollte durch Sparmaßnahmen in den öffentlichen Haushalten das Defizit innerhalb von vier Jahren halbieren. Die in 2010 neu gewählte Regierung einer Koalition aus Konservativen und Liberalen verschärfte das Tempo und will das Defizit bis 2014 beseitigen, was noch massivere Kürzungen zur Folge hat. Dabei werden
Renten und die Ausgaben für Gesundheit noch relativ geschont, vielmehr tragen der Bildungssektor und andere Sozialleistungen die Hauptlast, die den britischen Wohlfahrtsstaat radikal verändern wird. ${ }^{2}$

Die folgenden Ausführungen untersuchen, ob die 2010 angekündigten Reformen des englischen National Health Service (NHS) einen Paradigmenwechsel der seit dem Ende des 2. Weltkrieges verfolgten Gesundheitspolitik darstellt, oder ob es sich um eine Weiterentwicklung des schon vorher von den Konservativen eingeschlagenen und von der Labour-Regierung fortgesetzten Weges handelt, den strikt vertikal organisierten und von London gesteuerten NHS durch Formen des Wettbewerbs zu verändern. ${ }^{3}$ Meine Hypothese ist, dass gegenwärtig das Ziel der Haushaltskonsolidierung dominiert und dies der Anlass ist, neue Strukturen zu schaffen. Dahinter steht

1 Krugman 2012, Blog vom 27.1.2012

2 Hills, 2011, S. $38 \mathrm{f}$ 
die Überzeugung der Koalitionsregierung, dass Wettbewerb das beste Instrument ist, um in der medizinischen Versorgung hohe Qualität zu geringen Kosten zu erreichen

Die öffentliche Diskussion ist stark polarisiert, aber auch die medizinischen Fachgesellschaften wie die British Medical Association oder die Royal Colleges of General Practioners sprechen sich gegen die von der konservativ-liberalen Koalition angestrebten Reformen des NHS aus. ${ }^{4}$ Der »Daily Telegraph « titelte am 9. Juli 2010 nach der Vorlage des White Papers »Equity and Excellence: Liberating the NHS « durch den Gesundheitsminister: »Biggest Revolution in the NHS for 6o years«. Eine Gruppe von 400 Ärzten, anderen Heilberuflern und Gesundheitswissenschaftlern appellierten an das House of Lords, dem der Gesetzentwurf des House of Commons im Herbst 2011 zur Stellungnahme vorgelegt wurde: "It's time to kill the bill «. ${ }^{5}$ Nicht gerade akademischer Sprachgebrauch, aber ein deutlicher Hinweis, wie stark die Gesundheitsreform die Professionellen beschäftigt. Die englische Öffentlichkeit ist auch deshalb besonders sensibel gegenüber Versprechungen, durch mehr Wettbewerb und Privatisierung würde eine bessere Versorgung erreicht, weil sie am Beispiel der britischen Eisenbahnen das Gegenteil erlebt hat.

Um die von der konservativ-liberalen Koalition angestrebte Reform des NHS beurteilen zu können, sollen zunächst die mit der Gründung des NHS verfolgten Werte dargestellt werden. Diese werden von keiner Partei in Frage gestellt, aber die angestrebten Reformen sind daran zu messen. Es wird dargestellt, wie der NHS vor der Reform organisiert war. Das bildet die Basis, um die Reformelemente des neuen NHS zu analysieren und zu bewerten, ob dadurch Verbesserungen erreicht werden, oder die Gleichzeitigkeit von rigiden Sparmaßnahmen und organisatorischer Neuordnung dem System schaden könnte. Die Strukturen eines »befreiten « NHS werden daraufhin geprüft, was die Reformen absehbar bewirken und ob die selbst gesetzten Ziele erreicht werden, oder ob lediglich eine alte Bürokratie durch eine neue ersetzt wird, ohne dass Kosten, Qualität und Kontinuität der Versorgung besser werden. Entscheidend ist, welche Verhaltensanreize durch die neue Struktur des NHS gesetzt werden.
Private Leistungserbringung ist seit Gründung des NHS ein integraler Bestandteil. Es wird zu untersuchen sein, was sich seit den Reformen der siebziger Jahre verändert hat und ob die anstehende Reform noch einmal einen größeren Schub an Privatisierung bringen wird, so dass durch eine veränderte Quantität auch eine neue Qualität Platz greift. Zumindest ist das eine Sorge der Reformgegner, wobei Privatisierung häufig sprachlich ungenau als eine Metapher für eine zunehmende Kommerzialisierung und Ökonomisierung des Gesundheitssystems verwendet wird, die das berufliche Ethos der Professionellen im NHS, nur im Dienste der Patienten zu handeln, untergräbt. ${ }^{6}$

\section{Drei Phasen der Reform des NHS}

\section{Dezentralisierung und Wett- bewerb während der Regie- rungszeit Thatcher und Major}

Die Konservativen haben seit Ende der siebziger Jahre grundlegende Reformen im NHS durchgesetzt, die auch von Labour in einem wichtigen Punkt beibehalten wurden. Der markanteste Einschnitt war ganz im Geiste des neoliberalen Mainstreams in der Wirtschaftspolitik, Elemente von Wettbewerb in den NHS einzuführen. Zwischen 1979 und 1990 war Premierministerin Margret Thatcher an der Spitze einer konservativen Regierung. Sie wurde als »Iron Lady» bezeichnet, weil sie mit großer Härte für Deregulierung der Märkte, für Reduzierung der Staatstätigkeit und für Wettbewerb als alle anderen Regulierungsformen überlegenes Prinzip eintrat, das sie auch im NHS durchsetzen wollte.

Die radikalste Änderung gegenüber der bisherigen, zentralen Steuerung des NHS war die Trennung der Leistungserstellung ("provider« wie General Practioners (Hausärzte) und Krankenhäuser) von der Entscheidung über die Verwendung (purchaser) des NHSBudgets durch regionale Gremien des NHS, die Gesundheitsleistungen bei den Leistungserbringern einkaufen. Das Verfahren der »internen Märkte« sollte ein für alle verbindlicher Wettbewerbsprozess sein, in dem die Leistungen ausgeschrieben werden und sich die Anbieter über Preis und Qualität bemühen müssen, beauftragt zu werden. Dieser "purchaser-provider-split « hat auch mehr Wettbewerb in das System gebracht, aber er ist nicht flächendeckend umgesetzt worden, weil dafür regional die Voraussetzungen fehlten, z. B. eine Vielzahl von Anbietern. Auch die

\section{Das NHS ist von einer sehr alstaatlichen zu einer entralen Organisation miert worden.}

Dauer der Vergaben der Versorgungsaufträge schränkt die Möglichkeiten des Wettbewerbs ein, weil Krankenhäuser Investitionen erfordern, die lange Amortisationszeiten haben und die regionale Gesundheitsversorgung gewährleistet sein muss, auch wenn ein Krankenhaus oder eine Arztpraxis unwirtschaftlich arbeitet. Marktmechanismen sind nicht schlicht auf Güter der Daseinsvorsorge übertragbar. $^{7}$

Seit seiner Gründung ist der NHS häufig reformiert worden. Von einer sehr zentralstaatlichen Organisationen, die den Patienten wenig Wahlmöglichkeiten bei der Auswahl von Ärzten und Krankenhäusern ermöglichte, hat sich der NHS dezentralisiert, indem mehr Entscheidungsbefugnisse in regionale Gremien gegeben wurden, und der $\mathrm{Pa}$ tient nicht mehr auf seinen im Wohnbe-

\footnotetext{
3 In 1999 wurde im Rahmen einer staatlichen Neuordnung den Parlamenten von Schottland, Wales und Nordirland eine größere Autonomie zugestanden. Sie entscheiden in ihrem Bereich über ihren jeweiligen National Health Service (NHS), so dass im folgenden Text nur die Reform des NHS in England analysiert wird. Die anderen Landesteile sind davon nicht betroffen und planen auch keine vergleichbaren Reformen.

4 BMA, 2011 und Royal College of General Practioners, 2012

5 Marmot et al., 2011. Die Lords können eine Gesetzgebung durch das House of Commons nicht verhindern, aber durch ein aufschiebendes Veto bis zu einem Jahr verzögern. Das House of Lords hat im März 2012 der Gesetzesvorlage des House of Commons zugestimmt, so dass nunmehr gültig ist.

6 Marmot et al., 2010: »The Bill will do irreparable harm to the NHS, to individual patients, and the society as a whole. It ushers in a degree of marketization and commercialization that will fragment patient cae; aggravate risks to individual patient safety; erode medical ethics and trust within the health system; widen health inequalities; waste much money..."

7 Ham, 1999, S. 27ff
} 
zirk zuständigen Hausarzt (GP) angewiesen war, sondern er konnte seinen Arzt wählen, war dann aber in der Regel für ein Jahr an diese Entscheidung gebunden. Auch Krankenhäuser können die Engländer selber auswählen, wobei abgesehen von Spezialbehandlungen aber die räumliche Nähe zum Wohnort den Ausschlag geben dürfte, so dass die Möglichkeit der freien Wahl in vielen Regionen, wo es nur ein Krankenhaus gibt, faktisch eine geringe Rolle spielt. Dezentralisierung, mehr Wahlmöglichkeiten für Patienten und größere wirtschaftliche Autonomie für die öffentlichen Krankenhäuser durch Bildung von NHS Trusts (aus dem Haushalt ausgegliederte Einrichtungen mit größerer Selbständigkeit) und NHS Foundation Trusts (wie Trusts, aber noch weitergehende Freiheiten bei der Finanzierung von Investitionen) veränderten den NHS, ohne seine Grundprinzipien in Frage zu stellen.

Unter Premier John Major (19901997) blieb der Reformansatz, den NHS durch mehr Wettbewerb effizienter zu machen, erhalten. Die Regierung entwickelte dazu ein neues Instrument, nämlich GP-Fundholders. Das waren Gruppen von GPs, die mit dem NHS ein eigenes Budget verhandelten, aus dem sie die eigenen Leistungen finanzierten, aber auch stationäre Aufenthalte ihrer Patienten. Erwirtschaftete Überschüsse konnten zur Modernisierung der Praxen verwendet werden, durften aber nicht als Honorare ausgeschüttet werden. ${ }^{8}$

Damit war ein Mechanismus etabliert, medizinisch unnötige Überweisungen zum Facharzt oder ins Krankenhaus zu vermeiden. Das Bezahlsystem basierte auf Kopfpauschalen für die in das Modell eingeschriebenen Patienten. Die Teilnahme an den GP-FundholderModellen war für Ärzte und Patienten freiwillig. Es gab »Stop-Loss-Regeln«, nach denen der NHS Hochkostenfälle direkt bezahlte, so dass das Morbiditätsrisiko, was im Grundsatz von den GP-Fundholders getragen wurde, begrenzt war. Auch aus einem finanziellen Interesse bestand bei den beteiligten Ärzten ein Anreiz, Prävention und Rehabilitation zu stärken und gut mit Krankenhäuser zu kooperieren, denn gesunde Patienten sind in diesem Modell ein Vorteil. Die Vorzüge einer integrierten Versorgung können durch dieses wettbewerbliche Anreizsystem zum Tra- gen kommen. Aber auch Gefahren sind vorhanden, die dem Anspruch des NHS auf gleiche Versorgung für alle Bürger widersprechen. Es besteht ein Anreiz zur Risikoselektion, indem man die relativ gesunden Patienten umwirbt und an die eigene Praxis bindet.

Das Versprechen, die Patienten durch alle Versorgungsstufen $\mathrm{zu}$ betreuen, wurde auch eingelöst, aber mit einem Ergebnis, dass dem Selbstverständnis des NHS widersprach: Die Patienten der GP-Fundholders hatten kürzere Wartezeiten bei der Krankenhausbehandlung, weil die GP-Fundholders ihre Kaufkraft als Druckmittel einsetzen konnten. Es widersprach dem Selbstverständnis des NHS, den Zugang zur Versorgung ausschließlich vom medizinischen Bedarf abhängig zu machen, aber nicht von der Kaufkraft der Patienten. Die Disziplin bei der Bildung von Schlangen ist bekanntermaßen Teil der nationalen Mentalität, deshalb wurde das Überspringen von Wartezeiten bei der stationären Behandlung aufgrund finanzieller Motive als ungerecht empfunden. Allerdings war der Zeitraum, in dem die »internen Märkte« wirken konnten, zu gering, um empirisch belastbare Aussagen zu machen. ${ }^{9}$ Die jetzt von der konservativliberalen Koalition angestrebte Reform des NHS wird noch stärker als das GP-Fundholder Modell mit dem Problem konfrontiert sein, dass eine größere Wahlfreiheit und Wettbewerb einen Anreiz zu Risikoselektion bei den Patienten setzen, indem man sich auf relativ Gesunde konzentriert. Das kann die Ungleichheit in der medizinischen Versorgung erhöhen.

\section{Mehr Geld, Qualitätsziele und Kooperation statt Wettbewerb unter Labour}

Labour behielt die Trennung zwischen Einkaufsfunktion und Leistungserstellung, die unter den Konservativen als Voraussetzung für mehr Wettbewerb eingeführt wurde, bei. Sie schafften allerdings das Modell des GP-Fundholders nach der Regierungsübernahme 1997 ab, weil es in ihren Augen ein Verstoß gegen das Prinzip einer egalitären Versorgung war. Es änderte sich auch die Rhetorik, mit der Reformen des NHS begründet wurden: Nicht mehr Wettbe-

werb und Markt waren das Ziel, sondern Kooperation der Leistungserbringer, um eine bessere Qualität der Versorgung zu erreichen. ${ }^{10}$ Die Entscheidungsstrukturen sollten so verändert werden, dass die regionalen und lokalen Einheiten mehr Kompetenzen erhielten, über das vom Gesundheitsministerium zugewiesene Budget zu entscheiden.

Die Feinsteuerung des NHS nach den Labour-Reformen fand lokal statt, also am Ort der Leistungserstellung durch zunächst 350, dann 151 »Primary Care Trusts« (PCTs) statt. Damit lagen Planung der regionalen medizinischen Bedarfe und die Vergabe von Versorgungsaufträgen in einer Hand. Dieses »Einkaufsmodell« (Purchasing) für medizinische Leistungen wird in England auch als »Commissioning " bezeichnet (Oxford English Dictionary: etwas in Auftrag geben oder etwas zum Laufen bringen). Es stellt ein Instrument dar, durch »interne Märkte« Formen des Wettbewerbs in das Gesundheitssystem zu bringen, das im Grundsatz nicht wettbewerblich im Sinne von Steuerung durch Preise und Gewinnorientierung organisiert ist. Hier hat die Labour-

Die Feinsteuerung des NHS fand am Ort der Leistungserstellung statt.

Regierung den von den Konservativen eingeschlagenen Pfad fortgesetzt. Commissioning trat an die Stelle einer direkten Zuweisung der Mittel über den Haushalt und war eine Form der Deregulierung, die gleichzeitig aber neue Instrumente und Bürokratien zur Folge hat, um diesen Wettbewerb zu regulieren, damit die gesundheitspolitischen Ziele als Teil der öffentlichen Daseinsvorsorge erfüllt werden. Labour wollte mit Commissioning eine bessere Integration der ambulanten und stationären Versorgung erreichen, aber auch eine intensivere $\mathrm{Zu}$ sammenarbeit mit den sozialen Diensten der Kommunen (insb. Langzeitpflege und Betreuung psychisch Kranker), um die Kontinuität von Behandlungsprozessen zu erhöhen und damit Effizienz und Qualität verbessern.

8 Ham, 1999, S. 40ff

9 Le Grand, Mays, Mulligan, 1998

10 Ham, 1999, S. 51ff 


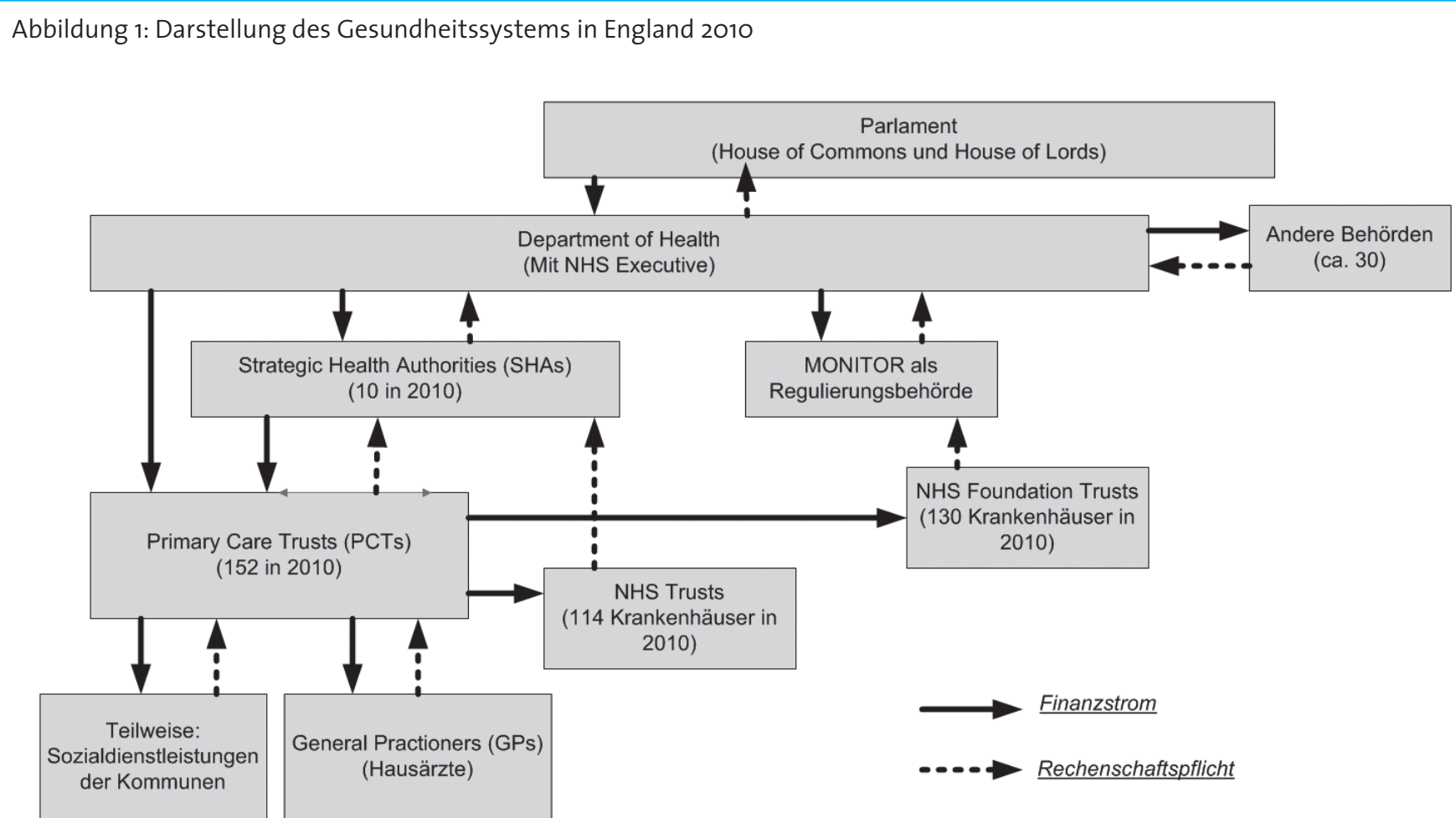

Quelle: Nach Harker, 2011, S. 11

Mit der Dezentralisierung verbunden war eine stärkere Steuerung über Ziele und eine intensivere Kontrolle der Qualität der Leistungen. Dafür wurden spezielle Organisationen geschaffen, die operativ von der Regierung unabhängig waren, aber doch eng politisch geführt wurden (»Arm's-length bodies«). Die Zahl der dieser Institutionen war mit mehr als 30 verwirrend groß, ihre Bezeichnung und Aufgabenstellung änderten sich im Zeitablauf und unterschiedliche Kompetenzen, die unklar abgegrenzt waren, führten auch zu Doppelarbeit. ${ }^{11} \mathrm{Zu}$ den wichtigen Organisationen gehörten u. a.:

- Seit 1999 NICE (National Institute of Clinical Excellence), was zuständig für medizinische Leitlinien und Kosten-Nutzen-Bewertungen von Arzneimitteln und medizinischen Prozeduren wurde.

- Seit 2009 das CQC (Care Quality Commission), indem seitdem die Funktionen der Qualitätssicherung im NHS vereinigt sind, die vorher von drei getrennten Organisationen wahrgenommen wurden.

- Die HPC (Health Protection Agency), die für Programme im Bereich Public Health zuständig war.
- MONITOR als Organisation, die für die Regulierung der Krankenhäuser in der Form von NHS Foundation Trusts zuständig war (Lizenzierung und finanzielle Überwachung).

Am Beispiel von MONITOR als Regulierungsbehörde, die die verselbständigten Krankenhäuser lizenziert und ihr finanzielles Gebaren kontrolliert, wird der Konflikt deutlich, der aus der stärkeren Wettbewerbsorientierung folgt. Die aus Qualitäts- und Effizienzgründen gewünschte stärkere Integration von Leistungserbringern erhöht auch die Gefahr, dass sie auf regionalen Märkten eine starke Marktmacht erhalten, die wettbewerbswidriges Verhalten begünstigt und die den PCTs zugedachte Führungsrolle bei der Aufteilung der Ressourcen durch Commissioning durch die PCTs in Frage stellen kann. ${ }^{12}$

Das insgesamt für den NHS verfügbare Budget wird zwar zentral entschieden und verteilt, aber das Gesundheitsministerium hat landesweit zehn regionale Unterorganisationen, die »Special Health Authorities « (SHA), deren Aufgabe darin besteht, das Budget den heute 151 lokalen Organisationen zuzuweisen, den »Primary Care Trusts « (PCTs), davon haben sechs zusätzlich die Aufgabe, auch die kommunalen Gesundheitsleistungen zu beauftragen, was am klarsten der Vorstellung einer auf den Patienten zentrierten, integrierten Versorgung entspricht. ${ }^{13}$

Die von Labour eingeführten PCTs waren das innovativste Element im NHS, weil sie das in der Höhe zentral vorgegebene Budget auf die lokalen Leistungserbringer im ambulanten und stationären Bereich verteilen, also für »Commissioning « verantwortlich sind. Sie entscheiden damit über ca. $80 \%$ der Ausgaben im NHS und die Entscheidung fällt dort, wo das medizinische Geschehen stattfindet, nämlich überwiegend lokal. ${ }^{14}$ Sie decken eine definierte Region ab, die um die 340.000 Einwohner umfasst, so dass eine ausreichende Größe erreicht ist, um Risiken zu poolen. ${ }^{15}$ Die Zuweisung des Budgets erfolgt einwohnerbezogen, wobei mit der Morbidität gewichtet wird und die regionale Kostenstruktur ebenfalls berücksichtigt wird.

Über das verbleibende Fünftel des Gesundheitsbudgets wird zentral ver-

\footnotetext{
11 Boyle, 2011, S.21ff

12 Garber, 2011, S. 16ff1

13 Boyle, 2011, S. 22f

14 Harker, 2011, S. 9

15 Boyle, 2011, S. 358
} 
fügt, um insbesondere medizinische Spezialversorgung sicherzustellen, die lokal wirtschaftlich nicht vorgehalten werden kann. Die PCTs müssen die medizinischen Bedarfe in der Region abschätzen und mit ambulanten und stationären Leistungserbringern Verträge abschließen, die ein entsprechendes Angebot sichern. Sie sind allerdings sehr stark an gesundheitspolitische Prioritäten gebunden, die zentral formuliert werden (z. B. Senkung der Wartezeiten oder andere, spezielle Programme wie Krebsbekämpfung), so dass ihre Autonomie begrenzt ist. Krankenhaustarife als Fixpreise auf der Basis von Kostenschätzungen und Arzthonorare sind ebenfalls zentral reguliert, aber die PCTs sollten durch ihre Einkaufsmacht die Versorgung lokal steuern, was aber nur begrenzt erfolgreich war. Sie müssen nicht mit allen Leistungserbringern Verträge abschließen, so dass auf Anbieterseite ein Wettbewerb entstehen kann. Das ist allerdings von den lokalen Bedingungen abhängig, denn wenn es lokal nur ein Krankenhaus gibt, ist die Entscheidungsfreiheit gering. 2006 wurde die Zahl der PCTs auf 151 halbiert, um ihre Handlungskompetenz durch größere Einheiten zu erhöhen. Dabei spielte auch eine Rolle, dass die PCTs die hohen Erwartung nicht erfüllt hatten, die Gesundheitsversorgung im Hinblick auf Qualität und Wirtschaftlichkeit zu steuern und die zugedachte Führungsrolle bei der Neuverteilung der Ressourcen in Richtung stärkere Integration und Vorrang für ambulante Versorgung wahrzunehmen. ${ }^{16}$

Die PCTs werden in ihrer Tätigkeit (Einhaltung des Budgets und Durchsetzung der zentral formulierten Qualitätsziele) durch die SHAs überwacht, so dass letztlich eine Kontrolle durch die Regierung möglich ist, und zwar nicht über Detailsteuerung, sondern die Vorgabe von Zielen und die Überwachung der Zielerreichung. Kontrolle erfordert Bürokratie und ist von den Betroffenen auch nicht immer geliebt, aber sie ist ein Instrument, um die Qualität der Versorgung in öffentlicher Verantwortung zu sichern.

Die PCTs haben die Betroffenen der Gesundheitsversorgung zu Beteiligten und Entscheidern gemacht hat. Alle betroffenen Gruppen sind an den PCTs be- teiligt: Es setzt sich aus Vertretern der GPs, Fachärzte, Krankenhäuser und anderer Heilberufe zusammen, aber auch aus Nicht-Professionellen (»lay people«) und Vertreter der Gemeinde. ${ }^{17}$ Damit ist erreicht, dass Entscheidungen nicht nur aus der Perspektive eines einzelnen Sektors getroffen werden, sondern die die Behandlungs- und Pflegeprozesse einschließlich der kommunalen Dienste integriert betrachtet und gesteuert werden. Regionale Planung der medizinischen Bedarfe und Beauftragung von Leistungen liegen in einer Hand. Das Modell will die Kooperation der Professionellen stärken und ist damit in einem wichtigen Aspekt als ein Gegenmodell $\mathrm{zu}$ den NHS-Reformen während der Regierungszeit der Konservativen entwickelt worden, weil sie die Kooperation in den Mittelpunkt stellten.

Die Labour Regierung unter Premierminister Tony Blair (1997-2007) schaffte die GP-Fundholders wegen der selektiven Wirkung beim Zugang zum Krankenhaus wieder ab, aber sie behielt die Grundidee der Reformen unter Thatcher und Major bei, nämlich die Trennung zwischen der Einkaufsfunktion und der Leistungserbringung, die überhaupt erst Formen des Wettbewerbs ermöglicht, von denen bessere Ergebnisse erwartet wurden. Insofern ähnelt der Ansatz dem Reformansätzen in Deutschland: Wenn man die Gesetzlichen Krankenkassen als Einkäufer von Leistungen betrach-

\section{Um den enormen Investitions-} stau in den Krankenhäusern zu beseitigen, wurde in großem Umfang privates
Kapital mobilisiert.

tet, dann sollen Selektivverträge ein Mehr an Wettbewerb unter den Leistungserbringern bewirken. Aber auch in Deutschland klaffen Wunsch und Realität auseinander, weil die Auswahl der Leistungserbringer durch zentrale Regulierung stark begrenzt ist.

Das Hauptziel der Labour-Regierung war, das öffentliche Ärgernis der sehr langen Wartezeiten in den Krankenhäusern für elektive Eingriffe oder Termine beim Facharzt. Die Ausweitung der Kapazitäten des NHS stand deshalb im Vordergrund. Aber man war auch bereit, private Leistungsanbieter an der Versor- gung von NHS-Patienten zu beteiligen. Seit 2004 konnte Commissioning durch die PCTs auch mit privaten Krankenhäusern, privaten Ärztenetzen und gemeinnützige Organisationen erfolgen. Um den enormen Investitionsstau in den Krankenhäusern zu beseitigen, wurde in großem Umfang privates Kapital mobilisiert und der Schuldendienst aus den Budgets der PCTs bestritten. Die Rhetorik der NHS-Reformen unter Labour war stark gegen Privatisierung und Markt gerichtet, aber um die Situation der Patienten schnell zu verbessern, hat man sich sehr pragmatisch des privaten Sektors bedient, wo es nützlich war. ${ }^{18}$

Labour hatte bei Übernahme der Regierung 1997 sechs Ziele der Gesundheitsreform formuliert: ${ }^{19}$

- Gerechtigkeit beim Zugang zu Einrichtungen des NHS im ganzen Land, insbesondere drastische Verringerung der Wartezeiten

- Vorgabe nationaler Qualitätsstandards und Verantwortung für ihre Durchsetzung auf lokaler Ebene

- Partnerschaft und Kooperation im NHS und enge Zusammenarbeit mit den Kommunen

- Bürokratieabbau durch mehr Ergebniskontrolle

- Fokus auf Qualität und Patientenorientierung durch »Clinical Governance

- Wiederherstellung des Vertrauens in die Leistungsfähigkeit des NHS.

Es blieb nicht bei verbalen Beschreibungen, sondern die anspruchsvollen Ziele wurden auch weitgehend quantifiziert und mit einem Zeitplan versehen. So sollten innerhalb von zehn Jahren u. a. folgende Ziele erreicht werden: ${ }^{20}$

• 7.000 neue Betten in den Krankenhäusern

- Neubau von 100 Krankenhäuser

- Modernisierung von 3.000 GP-Praxen

- Einstellung von 10.000 neuen Ärzten und 20.000 Pflegepersonen

- Schaffung von 1.000 neuen Studienplätzen in der Medizin.

16 Garber, 2011, S. 16 und Boyle, 2011, S. 359

17 Ham, 1999, S. 58

18 Boyle, 2011, S. 361ff und S. 399f

19 Department of Health, 1997 und Boyle, 2011, S. 347f

20 Boyle, 2011, S.354f 
Diese quantifizierten Ziele wurden auch weitgehend erreicht. So wurden die Wartezeiten für elektive Medizin und Konsultationen bei Fachärzten und GPs dramatisch gesenkt und in vielen Indikationsbereichen erreichten die Behandlungsergebnisse ein vergleichbar hohes Niveau wie in anderen europäischen Staaten. ${ }^{21}$ Die Ziele wurden mit harten Vorgaben und enger Kontrolle von »oben« durchgesetzt, was die propagierte Entscheidungshoheit für die lokalen Gremien erheblich beschnitt, so dass das Ziel der Integration und Kooperation der NHS- Leistungserbringer und der gesundheitsbezogenen, kommunalen Dienstleister eher ein Wunsch blieb und 2007 zu einer erneuten Veränderungen an der organisatorischen Struktur führte (»World Class Commissioning «). ${ }^{22}$

Gleicher Zugang für alle Bürger gehört zu den Kernzielen des NHS. Das ist im Hinblick auf Wartezeiten in Krankenhäusern auch erreicht worden. Was nicht geschafft wurde, ist regionale und statusbedingte Ungleichheit zu verringern. Auch wenn der Gesundheitszustand der Bevölkerung sich insgesamt verbessert hat, gibt es weiterhin große Unterschiede in der Morbidität und Mortalität zwischen Bevölkerungsgruppen. Die sind durch die Instrumente der medizinischen Versorgung auch nur schwer zu bekämpfen, weil sie ihre Ursache überwiegend in den ökonomischen Verhältnissen und in dem gesundheitsbezogenen Verhalten der Individuen haben. ${ }^{23}$

Die eindrucksvollen Verbesserungen im NHS durch mehr Personal und Investitionen spiegeln sich in den Ausgaben für Gesundheit im Staatshaushalt. Innerhalb von zehn Jahren nach Übernahme der Regierung durch Labour hat sich das Budget für den englischen NHS von 36,6 Mrd. Pfund in 1998/99 auf 102,0 Mrd. Pfund mehr als verdoppelt, was die größte Steigerung in der Geschichte des NHS darstellt. ${ }^{24}$. Die Investitionen stiegen 1998/99 von 1,67 Mrd. Pfund auf 4,58 Mrd. in 2008/09. ${ }^{25}$ Der relative Anteil der Gesundheitsausgaben am Bruttoinlandseinkommen wuchs im gleichen Zeitraum von 5,3\% auf 8,5\% . Der Anteil der Gesundheitsausgaben am Gesamthaushalt ist unter Labour kontinuierlich gestiegen, wohingegen andere Ausgaben wie für Soziale Sicherung, Bildung und Verteidigung anteilig gesunken sind. ${ }^{26}$ Darin spiegelt sich die hohe politische Priorität für den NHS.

Ein erheblicher Teil der Ausgabensteigerung ist allerdings für höhere Gehälter im NHS ausgegeben worden, was auch dem Umstand geschuldet ist, dass zusätzliches Personal nur bei einer guten Bezahlung zu gewinnen ist. Wanless et al. schätzen, dass 43\% der Ausgabensteigerung im NHS zwischen 2002/03 und 2007/08 für höhere Löhne ausgegeben wurde, die Produktivität also nicht erhöht hat. ${ }^{27}$ Die angestrebten Effizienzgewinne im NHS sind empirisch nicht belegbar, im Gegenteil, die Gesamtproduktivität ist leicht gesunken. Allerdings ist die Bildung eines Gesamtindikators für Produktivität in der Gesundheitsversorgung methodisch schwierig und umstritten, was die Aussage relativiert. ${ }^{28}$

Insgesamt stand die Labour-Regierung am Ende ihrer Amtszeit mit ihren Reformen gut da. Die Zufriedenheit der Engländer mit ihrem NHS war deutlich gestiegen. ${ }^{29}$ Die medizinischen Qualitätskennziffern und der Ressourceneinsatz zeigten deutliche Verbesserungen, so dass England den Vergleich mit anderen europäischen Ländern nicht scheuen muss, auch wenn es wie überall Verbesserungsmöglichkeiten gibt. Für Labour war Wettbewerb nicht gewinnorientierter Marktwettbewerb, sondern ein Anreizsystem auf der Basis von Kooperation. Institutionell wurden dazu die lokalen PCTs geschaffen, die durch die regionalen SHAs letztlich durch das Ministerium für Gesundheit kontrolliert wurden. Die größere betriebliche Unabhängigkeit der NHS-Krankenhäuser wurde beibehalten, um wirtschaftliche Verhaltensweisen anzureizen.

Die Instrumente der zentralen Zielvorgaben und Qualitätskontrolle wurden aber weiter ausgebaut, quasi als Gegengewicht zur größeren Unabhängigkeit der regionalen Entscheidungsgremien und Krankenhäuser. Die politisch beschlossenen Ziele auf nationaler Ebene wollte man auch lokal durchsetzen und hat dafür die entsprechenden Instrumente geschaffen. Bei den Leistungserbringern wurde das zum Teil als eine zu enge Kontrolle empfunden und zugespitzt als »targets and terror « kritisiert. Die Vielzahl der Institutionen, die den regionalen Entscheidern Vorgaben machen und die Zielerfüllung kontrollieren, waren aber auch der Grund für Kritik von Leistungserbringern und politischer Opposition, die durch zu viel Bürokra- tie eine Verschwendung von Ressourcen sahen, die besser für die Versorgung der Patienten eingesetzt werden sollten.

Realisiert wurden die Reformen der Labour-Regierung in einer Zeit mit wirtschaftlichem Wachstum, in der auch die öffentlichen Haushalte noch relativ ausgeglichen waren. Das änderte sich mit der Finanzkrise 2008 dramatisch. Die Folgen der ökonomischen Krise beherrschten die öffentliche Diskussion und Labour verlor im Mai 2010 bei den Parlamentswahlen die Mehrheit.

\section{Entbürokratisierung und Effizienz- steigerung als Ziel der Koalition aus Konservativen und Liberalen}

\section{Neue Strukturen des NHS England}

Nach den Neuwahlen zum Unterhaus im Mai 2010 stellte erstmals in der Geschichte des Vereinigten Königreichs eine Koalition aus Konservativen und Liberalen die Regierung. Schon im Juli 2010 hat der Gesundheitsminister »by Command of Her Majesty« (eine liebenswerte Formulierung aus Respekt vor der Tradition, inhaltlich hat die Königin nichts zu sagen) dem Parlament ein »White Paper « unter dem Titel »Equity and Excellence: Liberating the NHS zugestellt, in dem die Grundzüge der Reform des NHS beschrieben sind, die die neue Regierung anstrebt. ${ }^{30}$ Der Zeitplan sah ursprünglich vor, dass die Reformen bis 2013 umgesetzt werden sollten. Aber schon bei der Abstimmung über den Gesetzentwurf »Health and Social Care Bill« im Januar 2011 stimmte die Hälfte der liberalen Fraktion gegen den Antrag oder enthielt sich. Der Parteitag der Liberalen im Frühjahr 2011 verlangte einen Stopp des Verfahrens und eine intensive, öffentliche Diskussion der Reformpläne, zumal keine der Koalitionsparteien vor der Wahl weitreichende Reformen des NHS angekündigt hatten.
21 Boyle, 2011, S. 355ff
22 Boyle, 2011, S. 359
23 Acheson, 1998 und Boyle, 2011, S. 387f
24 Harker, 2011, Tabelle 2
25 Harker, 2011, S. 8
26 Nuffield Trust, 2011
27 Boyle, 2011, S. 396
28 Boyle, 2011, S. $400 f$
29 National Centre of Social Research, 2011 S. 141
30 Department of Health, 2010a 
Aufgrund der vielfältigen Kritik am Gesetzentwurf gab es ein öffentliches Anhörverfahren aller Interessengruppen (»Listening Exercise«), das im Juni 2011 beendet wurde. Organisiert wurde der Prozess vom »NHS Future Forum«, eine unabhängige Organisation, die aber der Regierung nahe steht. ${ }^{31}$ Ergebnis dieser Diskussionen waren inhaltliche Änderungen an dem Gesetzentwurf, der dann im Herbst 2011 dem »House of Lords " zugestellt und bis März 2012 beraten wurde. Die Regierung hat die Änderungsvorschläge des NHS Future Forum voll übernommen. ${ }^{32}$ Der ehrgeizige Zeitplan, dass der "neue " NHS im April 2013 seine Arbeit beginnen sollte, wurde aufgeweicht. Es wird auch eine Übergangszeit geben, in der alte und neue Institutionen nebeneinander existieren werden, was unter Kostenaspekten, aber auch im Hinblick auf die Klarheit von Entscheidungen nicht optimal ist. Die Lords haben das Gesetz im März 2012 in der Fassung des House of Commons beschlossen. Am 27. März 2012 hat die Königin ihre Zustimmung erklärte, was ein rein formaler Akt ist, so dass das die »Health and Social Care Bill« nunmehr Gesetz ist.

\section{Durch die Befreiung des NHS von falscher Regulierung sollen Gerechtigkeit und Exzellenz verwirklicht werden.}

Der Titel des White Paper »Liberating the NHS « vom Juli 2010 ist das Programm: Durch die Befreiung des NHS von falscher Regulierung sollen Gerechtigkeit und Exzellenz verwirklicht werden. Gerechtigkeit bezieht sich auf die Werte des NHS, dass jeder Bürger nach dem medizinischen Bedarf versorgt wird, nicht nach seiner finanziellen Leistungsfähigkeit. Im vom Premierminister, dem Vize-Premierminister von den Liberalen und dem Gesundheitsminister unterzeichneten Vorwort heißt es wörtlich, dass der NHS von einer überbordenden Bürokratie und der top-down Kontrolle befreit werden soll. Der NHS soll sich künftig gegenüber dem Patienten verantworten, nicht gegenüber einer Bürokratie. Der Patient soll mehr Wahlmöglichkeiten bekommen, wo oder durch wen er ambulante und stationäre Leistungen in Anspruch nimmt. Dazu soll er bessere
Informationen über die Ergebnisse medizinischer Behandlungen im Sinne von "Outcomes " bekommen, so dass er selber entscheiden kann, was zu ihm passt.

Wenn sich der Staat auf die Rolle beschränkt, das Gesamtbudget für Gesundheit festzulegen und auf die Leistungserbringer zu verteilen, die Einzelentscheidungen aber durch neue Verfahren ersetzt, in denen Ärzte entscheiden und der Patient letztlich durch seine Wahlentscheidung bestimmen soll, wer welche Leistung erbringt und bezahlt kriegt, dann liegt darin ein erheblicher Steuerungsoptimismus, dass dieser Prozess seitens der Leistungserbringer ohne egoistische Interessen funktioniert. Man kann auch daran zweifeln, dass der Patient aufgrund von Informationen über medizinische Ergebnisse seine Entscheidung trifft, welchen Leistungserbringer er wählt. Das mag heute wegen einer besserer Informiertheit der Patienten ein größere Rolle spielen als vor zwanzig Jahren, aber das Problem der asymmetrischen Information bleibt: Der Arzt weiß mehr über die richtige Diagnose und Therapie als der Patient und er entscheidet -wenn es gut läuft- im informierten Konsens mit dem Patienten. Je nach Bezahlsystem entscheidet er aber auch über sein Einkommen, was die Entscheidung beeinflussen kann. Der Patient hat ein Interesse, dass der Arzt in seinem Sinne entscheidet und die Qualität der Behandlung hinsichtlich Strukturen, Prozesse und Ergebnisse kontrolliert wird. Der Arzt "auf dem Fahrersitz", so die Formulierung des White Paper, dem vom Patienten auf dem Rücksitz gesagt kriegt, wohin die Fahrt gehen soll und von ihm kontrolliert wird, ob er alles richtig macht, ist zwar ein anschauliches Bild, aber fern der Wirklichkeit medizinischer Dienstleistungen. Der Arzt entscheidet in der Regel über Diagnose und Therapie.

Die Entscheidungen über die Verwendung der im Budget zugewiesenen Mittel sollte ursprünglich von den Hausärzten in »GP Consortia " getroffen werden, was als ein bottom-up Verfahren verstanden wurde, das an die Stelle der bisherigen, zentralen Steuerung treten sollte. Nach dem White Paper sollten das die GPs sein, weil sie als Hausärzte die größte Nähe zu ihren Patienten haben und ihre Interessen vertreten so die Idee. Die GPs sollten »auf dem Fahrersitz des NHS « künftig das System steuern, weil sie besser wissen, was für die Patienten gut ist.

Nach der öffentlichen Anhörung und Kritik an dieser Steuerung ausschließlich durch die GPs, ist in der Regierungsvorlage, die dem House of Lords im Herbst 2011 zur Stellungnahme zugeleitet wurde, das ursprüngliche Konzept der "GP-Consortia" zu einem »Clinical Commissioning Group « (CCG) verändert worden. Jetzt sollen auch mindestens ein Arzt aus der Sekundärversorgung in Krankenhäusern und eine Pflegekraft im »Governing Board « der CCGs beteiligt werden. Die Bezeichnung »Commissioning « ist mehr als eine kosmetische Änderung, weil dadurch die Aufgabe der CCGs betont wird, die Integration der Leistungserstellung über die Sektoren hinweg »zum Laufen zu bringen«. Das Grundkonzept bleibt aber: Der Staat nimmt sich zurück und vertraut nach dieser Vorstellung auf das dezentrale Verfahren des "Commissioning «, das überwiegend von Ärzten gesteuert wird. Dies ist Ausdruck der konservativen Kernüberzeugung, dass die Individuen besser als der Staat entscheiden können, wie welche Bedarfe gedeckt werden. Wenn schon nicht die Individuen selber, dann sollen zumindest die Ärzte auf lokaler Ebene entscheiden, die nach ihrem Ethos ausschließlich auf die Interessen der Patienten verpflichtet sein sollen. Das ist aber eine gewagte Abstraktion von tatsächlichen, ökonomischen Interessen, denn die CCGs, die die bisherigen PCTs und SHAs ersetzen sollen, entscheiden über die Verwendung von ca. $80 \%$ des NHS-Budgets, also über Ausstattung und Einkommen in der ambulanten und stationären Versorgung, aber damit auch über die Einkommen der Leistungserbringer in den betroffenen Einrichtungen. Damit sind Zielkonflikte zwischen dem medizinisch Vernünftigen und Einkommensinteressen programmiert. In der Presse wurden die Reformpläne für den NHS zum Teil als »Revolution « bewertet. ${ }^{33}$ Bezogen auf die CCGs ist es eher eine $»$ KonterRevolution «, weil die von den Konservativen unter Thatcher eingeführte Tren-

31 Department of Health, 2011 ${ }^{\mathrm{a}}$

32 House of Lords, 2011 und NHS Future Forum, 2011

33 Daily Telegraph vom 9. Juli 2010 


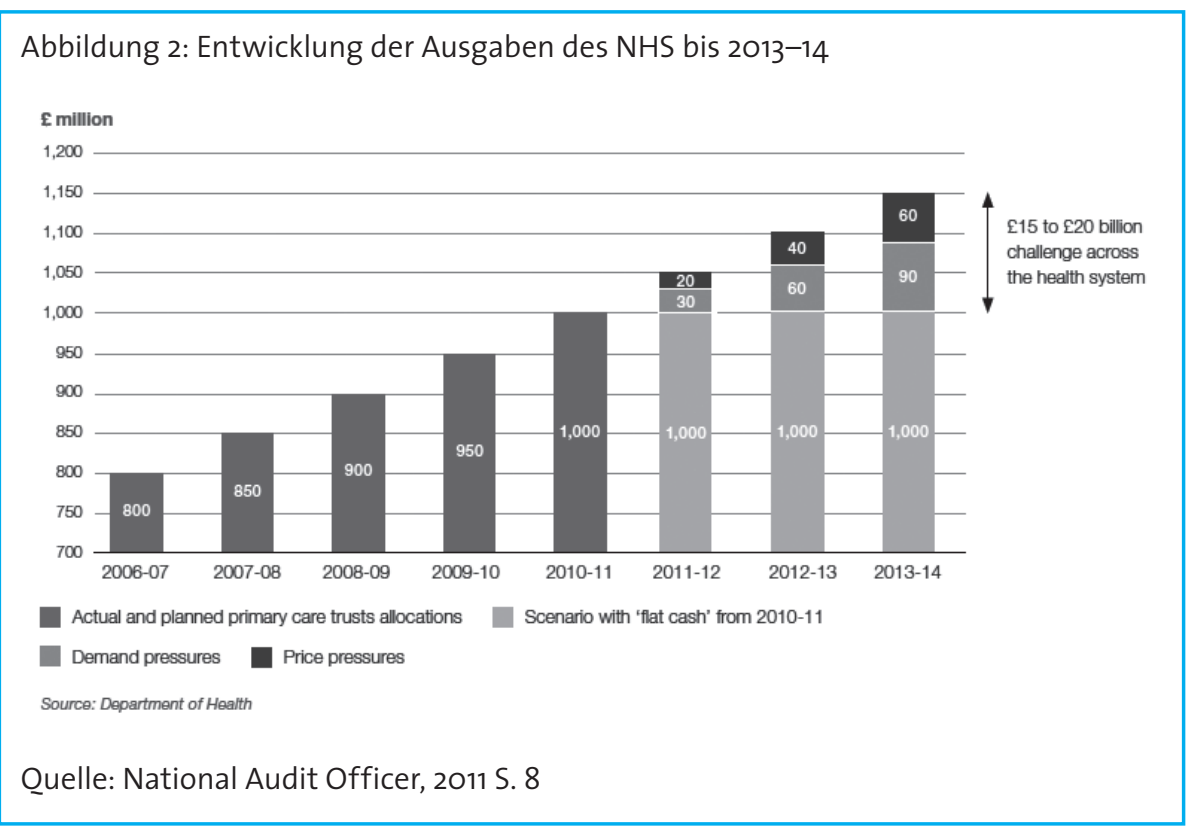

nung zwischen Einkaufsfunktion und Leistungserbringung, die Voraussetzung für mehr Wettbewerb sein sollte, wieder aufgehoben wird.

Die CCGs müssen in ihren Satzungen festlegen, welche Region sie versorgen wollen, was nicht mit der kommunalen Verwaltungsgliederung übereinstimmen muss. Medizinische Versorgung ist abgesehen von Spezialbehandlungen regionales oder lokales Geschehen. Künftig wird es verschiedene CCGs mit überlappender regionaler Zuständigkeit geben, was zu größerer Fragmentierung führt und es schwieriger mach wird, eine integrierte Versorgung zu organisieren. Mit der klaren, lokalen Zuordnung der PCTs war auch die Funktion verbunden, den lokalen Behandlungsbedarf zu planen und die Versorgung über die Vergabe der Einzelbudgets zu steuern, was bei populationsbezogenen Budgets, mit denen die CCGs ihre Leistungen einkaufen oder selber erbringen, nicht mehr möglich ist, sondern sich quasi im Selbstlauf durch das Nachfrageverhalten der Patienten ergeben soll. Die PCTs haben auch nicht nur über die im engeren Sinne medizinische Versorgung entschieden, sondern auch über einen Teil der "Community Care «, also der ambulanten Pflege in den Gemeinden. Das entfällt künftig und ein Teil des Budgets des NHS für »Public Health « in Höhe von einer Milliarde Pfund wird auf die Kommunen verlagert. Ob die Kommunen angesichts ihrer Haushaltsdefizite ihre Aufgaben in der öffentlichen Gesundheitsförderung allerdings künftig wahrnehmen können, ist angesichts der Krise der öffentlichen Haushalte zweifelhaft. Um die Verbindung zur kommunalen Versorgung herzustellen, werden mit den "Health and Well-Being Boards « als Teil der Gemeindeverwaltung neue Gremien geschaffen, die die Aktivitäten koordinieren sollen. Hier führt der versprochene Bürokratieabbau lediglich zu neuen Gremien, was auch unvermeidlich ist, weil die Schnittstelle mit den sozialen Diensten und Public-Health-Aufgaben in den Kommunen organisiert werden muss.

Nach der Revision des ersten Entwurfs der »Health and Social Care Bill« ist mehr Wettbewerb nicht mehr die erste Priorität der Regierung, aber er soll dadurch verstärkt werden, dass sich als Anbieter von ternehmen und Wohlfahrtsverbände um Budgets bei den CCGs bewerben können (»any willing providers «). ${ }^{34}$ Bisher werden nicht mehr als $2 \%$ der stationären Leistungen von Privaten erbracht. ${ }^{35}$ Die Mitgliedschaft der niedergelassenen Ärzte in einer CCG ist nicht mehr freiwillig wie beim Fundholder-Modell, sondern zwingend. In der Tendenz könnte damit eine weitere Privatisierung der Leistungserbringung im stationären Sektor verbunden sein, wobei die Leistungen selber weiterhin öffentlich, nämlich aus Mitteln des NHS erfolgt. Ob das angesichts der Budgetrestriktionen tatsächlich eine renditeträchtige AktiviGesundheitsleistungen auch private Un- tät für private Unternehmen sein wird, kann bezweifelt werden, zumindest wenn die Regulierung der Anbieterseite so erfolgt, dass eine "Rosinenpickerei ausgeschlossen ist. ${ }^{36}$ Dabei wird es entscheidend darauf ankommen, wie die Budgetzuweisung erfolgt, was bisher nicht klar erkennbar ist. Der kritische Faktor ist dabei, wie bei pauschalierten Budgets die unterschiedliche Morbidität der Patienten berücksichtigt wird, oder ob bei Krankenhausentgelten die Tarife auf der Basis von Kostenschätzungen die Realität richtig wiedergeben. Es sollen im Krankenhaus verbindliche Preise sein, also keine Höchstpreise, so dass im Grundsatz der Wettbewerb über die Qualität erfolgen sollte. Auf der Ebene der CCGs ist aber die tatsächliche Risikozusammensetzung ihrer Patienten entscheidend, ob die pauschalierten Zuweisungen in das von ihnen verwaltete Budget zu mehr oder weniger »Einkaufsmacht « führen, bzw. ob Gewinne oder Verluste entstehen. Daraus ergibt sich ein Anreiz, Patienten mit relativ guter Morbidität zu werben, was dem Anspruch des NHS, einen egalitären Zugang zur Versorgung zu gewährleisten, deutlich widersprechen würde. Der Wechsel von einem populationsbezogenen Ansatz des Commissioning durch die PCTs zu einem gruppenbezogenen durch die CCGs ist die radikalste Än-

\section{Wettbewerb ist nicht mehr die erste Priorität der Regierung, er soll aber dennoch verstärkt werden.}

derung in der Reform des NHS, weil sie falsche Anreize setzt.

Im Vergleich dazu wäre ein größerer Marktanteil für private Krankenhäuser weniger einschneidend, weil NHS Krankenhäuser und private Kliniken künftig der gleichen Regulierung unterliegen werden. Eine stärkere Tendenz zur Privatisierung von Dienstleistungen ist zu erwarten, weil sich die CCGs mit den komplexen Managementaufgaben überfordert sehen und private Dienstleister beauftragen werden. Dann könnte zwar

\footnotetext{
34 McKee, 2011, S. 1f

35 King's Fund, 2011, Myth Two

36 King's Fund, 2011, Myth Two
} 
der Abbau staatlicher Bürokratie als Erfolg gebucht werden, aber die dafür aufzuwenden Mittel gingen der Patientenversorgung verloren. Wenn man öffentliche Krankenhäuser in privates Management überführte und den Privaten freie Hand beim Personal gäbe und der Staat weiter für die Alterssicherung der bisherigen Beschäftigten verantwortlich bliebe, dafür gibt es bei der Krankenhausprivatisierung in Deutschland Beispiele, dann könnte die Privatisierung der englischen Krankenhausversorgung allerdings einen erheblichen Schub bekommen. Der Gesetzentwurf sagt dazu nichts. $^{37}$

\section{Effizienzgewinne zur Kompen- sation von Kostensteigerungen}

In der Allgemeinheit, wie die Ziele der Reform des NHS im »White Paper« und im Gesetzentwurf beschrieben sind, mag man nicht widersprechen: Der Patienten soll im Mittelpunkt stehen. Die im »White Paper« formulierte Forderung, dass keine Entscheidung ohne den Patienten getroffen wird (»no decision about me without me«) ist Teil der ärztlichen Ethik und sollte im ArztPatient-Verhältnis immer gelten. ${ }^{38} \mathrm{Da}$ mit gibt es aber keine Antwort darauf, wie der Konflikt über die Verwendung der Steuergelder gelöst werden kann, denn im Staatsbudget stehen viele Aufgaben in Konkurrenz zueinander. Im Vorwort des »White Paper« wird der Konflikt angesprochen: Angesichts der massiven Haushaltsdefizite und wachsender Staatschulden wären schwierige Entscheidungen (»difficult decisions«) zu treffen, die nicht am NHS vorbeigehen können. Es ist zwar geplant, dass die Ausgaben für Gesundheit in der Periode bis 2014/15 inflationsbereinigt insgesamt um $0,4 \%$ steigen sollen, was eine jährliche, nominale Steigerung von 1,3\% beinhaltet. Die Investitionsausgaben sollen um $17 \%$ sinken, die Verwaltungskosten sogar um 33\%. ${ }^{39}$ Die geringe reale Steigerung der laufenden Ausgaben ist keinesfalls ausreichend, wenn gleichzeitig die Anforderungen an den NHS durch eine älter werdende Bevölkerung und steigende Kosten durch den medizinischen Fortschritt zunehmen werden. ${ }^{40}$ Die dadurch absehbar steigenden Kosten sollen durch Einspa- rungen von 15-20 Milliarden Pfund erwirtschaftet werden (Abbildung 1)

Die große Herausforderung für den NHS besteht darin, den Anstieg der Gesundheitsausgaben zu kontrollieren, aber gleichzeitig einen hohen Standard der medizinischen Versorgung zu gewährleisten. Angesichts steigender Ausgaben wegen einer älter werdenden Bevölkerung, in der der Anteil chronisch Kranker zunimmt, Multimorbidität und dauernde Pflegebedürftigkeit verbunden mit Demenz bei den Hochbetagten steigen und der medizinische Fortschritt zu höheren Kosten führt, weil neue Diagnosemethoden und Therapien teurer werden, kommt das der Quadratur des Kreises gleich. Alle entwickelten Länder stehen vor diesem Problem, egal ob stark privatwirtschaftlich geprägt wie die USA, oder Sozialversicherungsländer wie Deutschland. Überall wird versucht, durch die Neuorganisation von

\section{Der NHS steht mit der beab- sichtigten Strukturreform und den Folgen der Haushaltskrise unter doppeltem Stress.}

Prozessen in der Versorgung mögliche Effizienzreserven zu mobilisieren, also die gesundheitspolitischen Ziele mit weniger Aufwand zu erreichen, oder, was realistischer ist, einen Teil der unvermeidbaren steigenden Ausgaben aus Umstrukturierungen zu refinanzieren. Überall steht im Fokus, den Behandlungsprozess nicht aus der Perspektive der einzelnen Sektoren des Versorgungssystems zu betrachten, sondern aus der Perspektive des Patienten, und dabei die Kontinuität der Behandlung mit hohen Anforderungen an die Qualität zu sichern.

Der NHS steht mit der beabsichtigten Reform seiner Strukturen und den Folgen der Haushaltskrise unter doppeltem Stress. Wie oben dargestellt, wird der Gesundheitssektor im Vergleich zu anderen Staatsaufgaben bevorzugt behandelt. Aber gleichzeitig steigen absehbar künftige Ausgaben des NHS durch eine älter werdende Bevölkerung und den medizinischen Fortschritt. Die Erwartung an den NHS ist, die Ausgabensteigerungen bei unveränderter Qualität der Versorgung durch Effizienzsteige- rungen aufzufangen. Schon 2009, also noch unter Labour, hat der Chef des NHS David Nicholson angesichts der Krise des öffentlichen Haushaltes das Ziel formuliert, bis 201520 Milliarden Pfund im NHS einzusparen. Das entspräche einer Effizienzsteigerung von jährlich rund $4 \%$. (in der öffentlichen Diskussion "Nicholson Challenge " bezeichnet) ${ }^{41}$ Der Gesundheitsausschuss des Unterhauses teilt diese Zielsetzung und lässt sich über den Fortschritt fortlaufend berichten. ${ }^{42}$ Premierminister David Cameron hatte das Ziel schon in seinem Wahlkampf mit der die Probleme verdeckende Formulierung unterstützt, er wolle das Defizit des NHS kürzen, aber nicht die Leistungen (»Ill cut the deficit, not the NHS «) $)^{43}$

Es bestehen aber erhebliche Zweifel, ob die mit der "Nicholson-Challenge" angestrebten Effizienzsteigerungen in einer Größenordnung von jährlich 4\% überhaupt erreichbar sind. Zwischen 1997 und 2008 ist die Produktivität im NHS jedenfalls jährlich um $0,2 \%$ gesunken, so dass die neue Ziellinie mehr als ehrgeizig ist, oder mit erheblichen Begrenzungen von Leistungen und/oder der Bezahlung von Krankenhäusern und GPs erkauft werden müsste. ${ }^{44}$ Schon heute häufen sich Berichte, dass die Leistungen wegen nicht ausreichender Budgets gekürzt werden. ${ }^{45}$ Die in der Öffentlichkeit und unter den Leistungserbringern und ihrer Verbänden geführte Diskussion über die Gesundheitsreform trennt nur zum Teil zwischen den beabsichtigten Strukturreformen und den befürchteten Restriktionen im Budget des NHS. Das ist auch nachvollziehbar, weil die Regierung durch die nach unten auf die Ärzte verlagerten Entscheidungen sich ein Stück aus der Verantwortung für die Folgen von Budgetkürzungen zurückzieht. Die Kritik, dass das Gesundheitsministerium die Verantwortung für den NHS abgeben würde, ist zwar

\section{Illiffe, 2012}

38 Department of Health, 2010a , S. 3

39 HM Treasury, 2010

40 Dixon, 2011, S. $52 \mathrm{f}$

41 Nicholson, 2009

42 House of Commons, Health Committee, 2011

43 The Guardian vom 17.10.11 http://www.guardian.co.uk/society/2011/oct/17/revealedtoll-cuts-nhs-services/print (20.2.12)

44 Phelps et al., 2010, S. 105

45 The Guardian vom 17.10.2011 
Abbildung 3: Struktur des NHS England nach der Reform

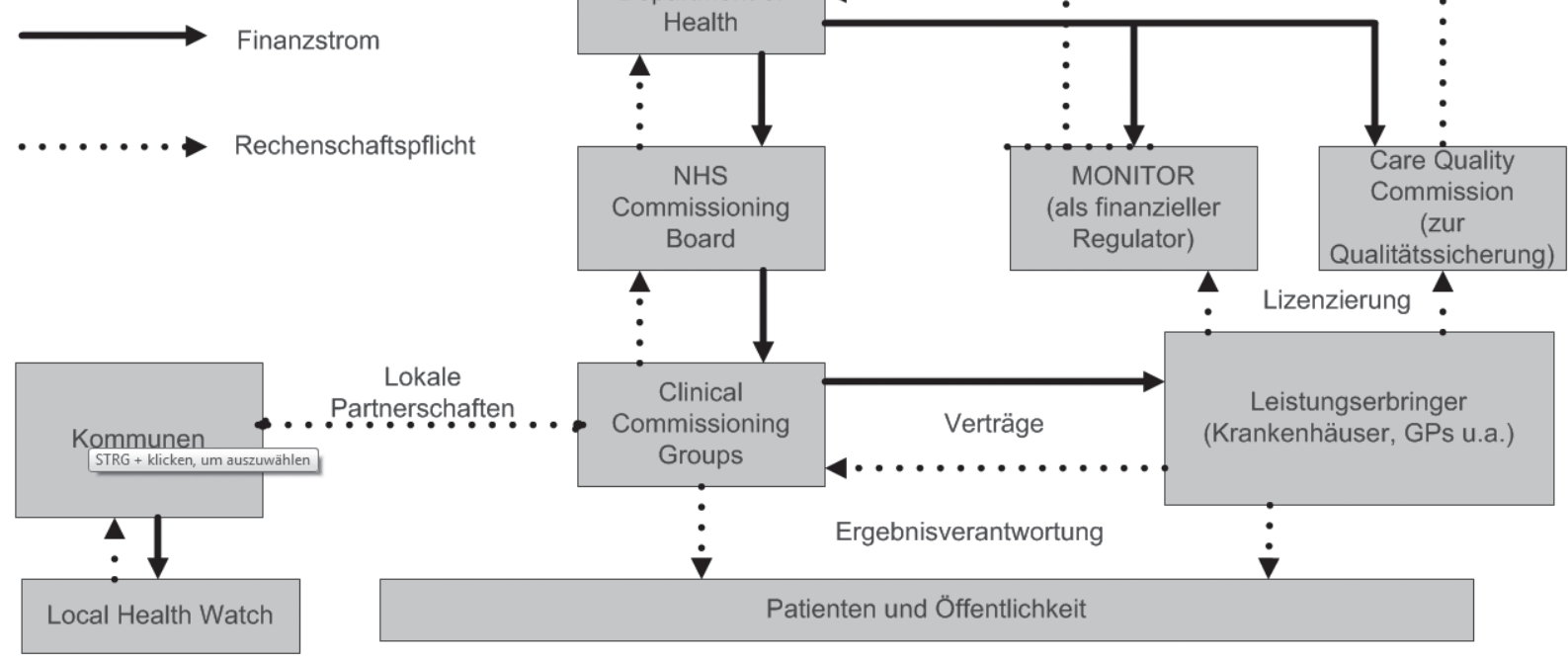

Quelle: Nach Department of Health, 2010, S. 39

von der Regierung bestritten worden; sie hat in ihrer geänderten Vorlage an das Oberhaus auch noch einmal betont, dass das Department of Health weiterhin die politische Verantwortung tragen würde und verantwortlich gemacht werden könne. Was das nun wiederum konkret bedeutet, ist nicht ersichtlich, weil die bisherigen Institutionen, nämlich die SHAs und die lokalen PCTs, abgeschafft werden sollen. Die Regierung erwartet durch diesen Abbau von Bürokratien eine Kostenersparnis von ca. 850 Millionen Pfund bis 2013/14. ${ }^{46}$ $\mathrm{Ob}$ das erreicht werden kann, ist zweifelhaft, denn auch die neu geschaffenen Institutionen, die an die Stelle der alten treten, werden einen Verwaltungsapparat benötigen. Auch die CCGs werden Managementkosten verursachen und nicht quasi nebenbei von den Ärzten betrieben werden können. In dem Maße, wie die Regierung rechenschaftspflichtig gegenüber dem Parlament bleibt, wird sie auch einen Apparat benötigen, um Qualität und Wirtschaftlichkeit des NHS kontrollieren zu können. Kritik an Bürokratiekosten ist zwar populär, aber die Öffentlichkeit will auch wissen, ob die Steuergelder effizient verwendet werden, deshalb braucht man Bürokratien, die steuern und kontrollieren.

\section{Neue Bürokratien}

An die Stelle der bisherigen Institutionen PCTs und SHAs treten zwei neue Behörden, die zwar ein hohes Maß an Selbständigkeit im Verhältnis zum Ministerium haben sollen, aber letztlich auch Regierung und Parlament verantwortlich sind. Das »NHS Commissioning Board « (NHSCB) soll künftig dafür zuständig sein, die Werte des NHS im Versorgungssystem zu wahren, das vom Parlament bewilligte Budget auf die CCGs zu verteilen, sie in ihrer Arbeit $\mathrm{zu}$ überwachen und $\mathrm{zu}$ unterstützen sowie die Qualität der Behandlung zu sichern. Für Qualitätssicherung soll es aber weiterhin eine besondere Agentur geben, und zwar die »Care Quality Commission «, wobei noch unklar ist, wie die Aufgaben gegeneinander abgegrenzt werden. ${ }^{47}$ Weiterhin ist das Board durch direktes »Commissioning « für medizinische Spezialdienste verantwortlich, also für rund $20 \%$ des NHS-Budgets. ${ }^{48}$ Die Regierungsagentur MONITOR war bisher für die Lizenzierung und Überwachung der Finanzen der Krankenhäuser (NHS-Trust und mit erweiterten Kompetenzen NHS
Foundation Trusts) zuständig. Künftig sollen alle Krankenhäuser »Foundation Trusts« werden, die von MONITOR überwacht werden. MONITOR ist künftig auch zuständig für die Preise, die im NHS für einzelne Leistungen verlangt werden dürfen. MONITOR hat also künftig die Rolle eines Regulators hat für Dienstleister im NHS und

Der Wechsel von einer populationsbezogenen Budgetzuweisung zu gruppenbezogenen Budgets ist das größte Risiko für die Zielerreichung des NHS.

private Leistungserbringer, so dass ein einheitliches Preis- und Qualitätssystem geschaffen wird. ${ }^{49}$ NHSCB und MONITOR sind einerseits Instrumente der öffentlichen Kontrolle, andererseits aber auch eine wesentliche Beschneidung der Entscheidungskompetenzen der dezent-

46 King's Fund, 2011, Myth Four

47 Dixon, 2011, S. 55

48 Department of Health ${ }^{\mathrm{b}}$ (2011)

49 Department of Health, 2012, S. $4 \mathrm{ff}$ 
ralen Leistungserbringer, weil sie Vorgaben machen können.

Insbesondere das NHSCB könnte sich zu einer Mammutbehörde entwickeln, die den NHS künftig steuert, weil dort die Entscheidungen über die Budgetzuteilung fallen und ein Eingriffsrecht besteht, wenn die Budgetvorgaben nicht eingehalten werden. Die schwierigste Aufgabe für das NHSCB liegt in der richtigen Bemessung des Budgets für die einzelnen CCGs. Sie müssen die tatsächliche Morbidität der bei den CCGs eingeschriebenen Patienten richtig widerspiegeln, sonst wird ein starker Anreiz für die CCGs gesetzt, eine Strategie der Risikodifferenzierung zu betreiben, die auf Patienten mit geringem Morbiditätsrisiko zielt, um die tatsächlichen Ausgaben unter den Budgetansätzen zu halten. Der Wechsel von einer populationsbezogenen Budgetzuweisung, wie sie zur Zeit noch bei den PCTs praktiziert wird, zu gruppenbezogenen Budgets für die CCGs, die nicht regional gebunden sind, sondern sich aufgrund der Wahlentscheidung der Patienten bilden, ist das größte Risiko für die Einhaltung des Ziels des NHS, allen Bürgern eine gleiche Versorgung zu garantieren. Das deutsche Krankenkassensystem ist ein gutes Beispiel, dass die 1993 eingeführte Wahlfreiheit des Versicherten zwar zu mehr Wettbewerb geführt hat, aber nicht zu einem Wettbewerb um bessere Leistungen bei vergleichbaren Beiträgen, sondern aus der Perspektive einer einzelnen Kasse war es sinnvoller, sich um Versicherte mit einer guten Morbidität zu bemühen. Der Risikostrukturausgleich unter den Kassen war die Antwort des Gesetzgebers auf unerwünschte Risikoselektion. Das Beispiel zeigt, dass das Problem theoretisch und praktisch lösbar ist, aber dass es auch ein weiter Weg ist, bis Verfahren gefunden sind, die tatsächliche Morbidität richtig abzubilden. Eine direkte Erfassung der Morbidität erfolgt in Deutschland erst seit 2009. Die sozio-demographischen Merkmale, die man in England bisher für die Bemessung der regionalen Budgets verwendet hat, werden nicht ausreichen, um den CCGs die »richtigen« Budgets zuzuweisen.

In den ursprünglichen Vorstellungen des »White Paper« war noch eine gewisse Logik erkennbar, da die zentrale Steuerung und Kontrolle zum Patienten verlagern werden sollte, was staatliche Institutionen erübrigen sollte. Aber schon darin lag der Denkfehler, dass Markt ohne Bürokratiekosten funktionieren kann. Bei den Ökonomen wird das etwas neutraler als Transaktionskosten benannt, aber in der Sache ist es das Gleiche. Um Verträge abschließen zu können, über die künftig das NHSBudget auf die Leistungserbringer verteilt werden soll, fallen zunächst Kosten der Information und für Verhandlungen an. Dann muss die Einhaltung der Verträge kontrolliert und bei Verstößen sanktioniert werden. Zum Nulltarif ist das nicht zu haben. Wenn die politische Kontrolle durch Parlament und Regierung nicht aufgegeben werden soll, welches demokratisch gewählte Parlament würde darauf verzichten wollen? - dann muss es auch eine Verwaltung geben, die das steuert und kontrolliert. Das Versprechen von Bürokratieabbau hört sich immer gut an, wenn damit die Abschaffung überflüssige Prozesse gemeint ist, ist das auch vernünftig. Aber bei den Reformen für den NHS ist ohne Ausformulierung der Details, die bisher nicht vorliegen, keine abschließende Bewertung möglich, ob nicht lediglich bisherige Institutionen durch andere ersetzt werden. Angesichts der großen

\section{Unverändert bleibt die}

\section{Steuerfinanzierung des NHS und eine weitgehende Freiheit von Zuzahlungen.}

Herausforderungen, die durch die finanziellen Restriktionen bedingt sind, wäre es wahrscheinlich sinnvoller, bestehende Institutionen weiterzuentwickeln, statt das vorhandene System umzubauen, ohne zu wissen, ob das neue funktioniert. $^{50}$

Im ursprünglichen Konzept der »GPConsortia « im »White Paper «, das dem von Labour abgeschafften System der GP-Fundholders nachgebildet war, wurde der GP als »Lotse « im Gesundheitssystem zum »Kapitän «, der die Entscheidungen trifft und nur dem Patienten gegenüber verantwortlich sein sollte. Mit der neuen Konstruktion des "Clinical Commissioning Groups « verlagert man die Interessenkonflikte durch die Beteiligung von Krankenhausärzten in die Entscheidungsgruppe. Wie sich diese Struk- turveränderung auswirkt, wird erst in der Praxis zu beurteilen sein, denn die Konstruktion ist widersprüchlich: Der Vertreter des Krankenhauses sitzt auf Seiten der CCGs am Verhandlungstisch, wenn stationäre Leistungen eingekauft werden. Das kann zu besserer Kooperation führen, aber auch zum Ausspielen von Marktmacht, wenn ein Krankenhaus in einer Region eine dominierende Stellung hat. Auf jeden Fall eine komplizierte Rollenverteilung, bei der unklar ist, in welche Richtung die Anreize gesetzt werden. Die Vorlage der »Health and Social Care Bill« an das Oberhaus betont, dass die Interessen der Patienten im Mittelpunkt stehen sollen, wozu die Integration der Versorgung und eine hohe Behandlungsqualität notwendig sind. Gleichzeitig ist aber klar, dass die Budgets der CCGs den steigenden Aufgaben nicht angepasst werden, so dass sie die von der Regierung angekündigten »harten Entscheidungen" werden treffen müssen. Dass dies nicht durch Leistungskürzungen, sondern dadurch erfolgt, dass Effizienzreserven erschlossen werden, ist zunächst einmal das Prinzip Hoffnung. Es gibt international Beispiele für eine höhere Wirtschaftlichkeit von gut integrierten Versorgungsmodellen, aber sie funktionieren nur, wenn alle Beteiligten ein hohes Interesse an $\mathrm{Zu}$ sammenarbeit haben und von den Vorteilen überzeugt sind, wofür die Freiwilligkeit der Maßnahme eine wichtige Voraussetzung ist, aber auch Managementqualitäten. ${ }^{51}$ In England sieht es nicht danach aus, dass die NHS-Reform bei den Leistungserbringern auf große Zustimmung stößt, eher trifft das Gegenteil zu, was keine gute Voraussetzung ist, um die schwierige Aufgabe bei der Konsolidierung des Gesundheitshaushaltes, die mittelfristig ansteht, zu lösen.

\section{Fazit}

Ob die beschlossenen Reformen eine Abkehr vom bisherigen Pfad des NHS sind, lässt zurzeit noch keine eindeutige Antwort zu. Dazu sind noch zu viele Fragen offen, wie im Detail die Entscheidungsverfahren aussehen werden. Die Regierung ist jedenfalls entschlossen,

50 Wilkinson, 2011, S. $114 \mathrm{f}$

51 Ham, Zollinger-Read, 2012, S. 189ff und McClellan et al., 2010, S. $982 \mathrm{ff}$ 
unter dem Druck des Defizits im Staatshaushalt die Zuweisungen an den NHS real nur geringfügig zu steigern. Zusätzliche Gesundheitsausgaben aufgrund einer geänderten Morbidität und des medizinischen Fortschritts muss der NHS aus Einsparungen finanzieren. $\mathrm{Ob}$ das ohne Leistungskürzungen angesichts der finanziellen Dimensionen erreichbar ist, kann bezweifelt werden, zumindest hat es Effizienzsteigerungen von jährlich $4 \%$ bisher im NHS nie gegeben. Was unverändert bleibt, ist die Steuerfinanzierung des NHS und eine weitgehende Freiheit von Zuzahlungen bei Inanspruchnahme von Leistungen, was ein wesentliches, wenn nicht das entscheidende Merkmal für den sozialen Charakter des NHS ist.

Wettbewerb und private Leistungserbringung sind keine neuen Elemente im NHS, sondern sie sind bereits von Konservativen eingeführt und unter Labour fortgesetzt worden. Neu ist allerdings der Umfang, weil »Commissioning « für alle Leistungserbringer verbindlich werden soll und alle, also private Leistungserbringer und NHS-Einrichtungen, ihre Dienste anbieten können. Die bisherigen

\section{Folgen der Konsolidierung im Krankenhaussektor}

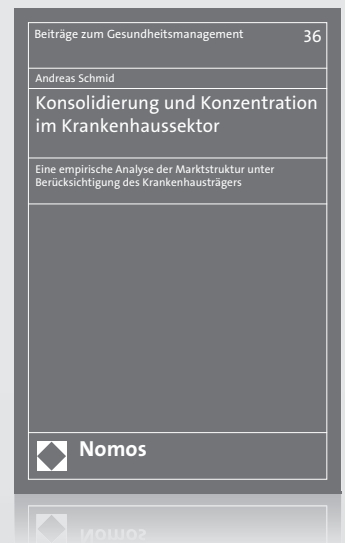

\section{Konsolidierung und Konzentration im Krankenhaussektor}

Eine empirische Analyse der Marktstruktur unter Berücksichtigung des Krankenhausträgers

Von Andreas Schmid

2012, 243 S., brosch., 38,- €, ISBN 978-3-8329-7595-1

(Beiträge zum Gesundheitsmanagement, $B d$. 36)

Durch die starke Konsolidierung im Krankenhaussektor droht in vielen Regionen die Bildung von Konzentration und Marktmacht. Dies kann für Patienten wie auch für Krankenkassen negative Folgen nach sich ziehen. Die Studie widmet sich der empirischen Erfassung des Konzentrationsprozesses und berücksichtigt dabei erstmals die Rolle der Krankenhausträger.

Weitere Informationen: www.nomos-shop.de/15231

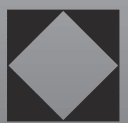

Entscheidungsverfahren über die lokalen Budgets des NHS sollen radikal dezentralisiert werden, mit der Gefahr, dass das »National« im NHS verloren geht und Gesundheitspolitik auf nationaler Ebene zu einer reinen Kürzungspolitik zu Lasten des NHS verkümmert. $\mathrm{Ob}$ das eintritt hängt davon ab, wie stark die neu geschaffenen Behörden NHSCB und MONITOR auf nationaler Ebene regulierend einwirken. Das wird nicht an den absoluten Ausgaben für Gesundheit zu sehen sein, die werden um knapp über $1 \%$ steigen, sondern ob die Mittel reichen, die wachsenden Aufgaben $\mathrm{zu}$ finanzieren. Deshalb ist die Kritik nachvollziehbar, die Organisationsreform unter der Überschrift einer Entbürokratisierung des NHS lenke von den Problemen ab, die wirklich gelöst werden müssen.

Die Abkehr von dem bevölkerungsbezogenen Bezug der Leistungserbringung hin zu einem gruppenbezogenen, in dem die »Clinical Commissioning Groups" für eine von ihnen betreute Gruppe von Patienten Leistungen erbringen, dürfte das schon jetzt im NHS bestehende Probleme der Ungleichheit im Gesundheitsstatus und in der regionalen Gesundheitsversorgung noch schwieriger lösbar machen. Das neue System setzt Anreize, dass sich die CCGs um gute Risiken bemühen, was zu Lasten der Patienten mit schlechter Morbidität gehen könnte.

Eine stärkere Integration der verschiedenen Sektoren des Gesundheitssystems hat die Chance, die Qualität zu verbessern und die Kosten zu senken, aber das ist nicht in erster Linie von den neuen Strukturen im NHS abhängig, sondern von dem materiellen Anreizsystem und dem Engagement der beteiligten Leistungserbringer. Die Anreize durch das neue Gesetz lassen aber eher befürchten, dass die Fragmentierung in der Versorgung zunimmt. Die Sorge ist, dass alte Bürokratien durch neue ersetzt werden und der angekündigte Kompetenzzuwachs für die Ärzte, die auf dem "Fahrersitz des NHS « Platz nehmen sollen, sich als eine Strategie erweist, dass sie für Kürzungen von Leistungen verantwortlich gemacht werden, die aus unzureichenden Budgetzuweisungen resultieren.

Literatur ist beim Autor erhältlich 